\title{
INTERFEROMETRIC PROCESSING OF SLC SENTINEL-1 TOPS DATA
}

\author{
Raphaël Grandin ${ }^{(1)}$ \\ ${ }^{(1)}$ Institut de Physique du Globe de Paris, Sorbonne Paris Cité, Univ Paris Diderot, CNRS, F-75005 Paris, France, Email: \\ grandin@ipgp.fr
}

\section{ABSTRACT}

InSAR processing usually involves two successive steps: focusing and interferometry. Most public-domain InSAR processing toolboxes are capable of performing both operations with data acquired in the standard Stripmap mode, starting from raw SAR data (level 0). However, the focusing of burst-mode data, such as TOPS and ScanSAR, requires substantial modifications to standard focusing methods due to the particular spectral properties of these data. Anticipating on this potential difficulty for non-expert users, the European Space Agency has chosen to release Sentinel-1 TOPS data in a Single Look Complex format (level 1). The data are already focused using state-of-the-art processing techniques, with phase information preserved. Even so, the focusing method introduces an additional quadratic phase term in the azimuth direction. In case of a small misregistration error between a pair of images, this residual term leads to steep phase ramps in azimuth that are superimposed on the desired interferometric phase. Therefore, this quadratic phase term needs to be removed from the SLC data prior to interferogram calculation. Here, a pre-processing method allowing for compensating this phase term and simply feeding the corrected SLC data into a standard InSAR processing chain is described. The method consists of three steps. The first step uses the metadata in order to reconstruct a continuous image in the azimuth direction, accounting for the small overlap between adjacent bursts ("stitching"). In the second step, multiplication of the images by an appropriate phase screen is performed so as to cancel the azimuthal quadratic phase term ("deramping"). The deramping operation uses the metadata, as well as the azimuth time lag between the images deduced from sub-pixel image correlation, in order to determine small misregistration errors. Misregistration errors are compensated using a simple affine relation deduced from least-square fitting of the azimuth offsets. Following this second step, the azimuth phase ramps are significantly reduced in the corrected interferogram. The third step consists in refining the affine coefficients that account for the misregistration error. The refinement is achieved by differencing the backward- and forward-looking interferograms, exploiting the spectral diversity in burst overlap regions ("spectral diversity"). This final step makes it possible to remove residual phase jumps across burst boundaries with the desired level of accuracy.
Key words: Sentinel-1 ; TOPS ; burst synchronization ; coregistration ; spectral diversity.

\section{WIDE-SWATH BURST-MODE DATA}

Azimuth spectral properties of burst-mode SAR data, such as ScanSAR [1] or TOPS [2], are significantly different from those of Stripmap. In burst-mode, the system observes a series of sub-swaths by periodically steering of the antenna in the elevation direction and transmitting a short succession of pulses gathered in the so-called "bursts". As a consequence, a given target is imaged only during a fraction of the equivalent stripmap synthetic aperture duration, thereby reducing its duration of illumination. Therefore, the increased swath width comes at the expense of azimuth resolution (Fig. 1).

Furthermore, the Doppler centroid of targets within any burst becomes variable in azimuth. Due to the latter effect, the impulse response function of standard burstmode focusing methods includes a residual phase term that exhibits a characteristic quadratic variation of the azimuth phase. This phase term needs to be compensated prior to using the phase for interferometric applications, a process referred to as "deramping" $[3,4]$.

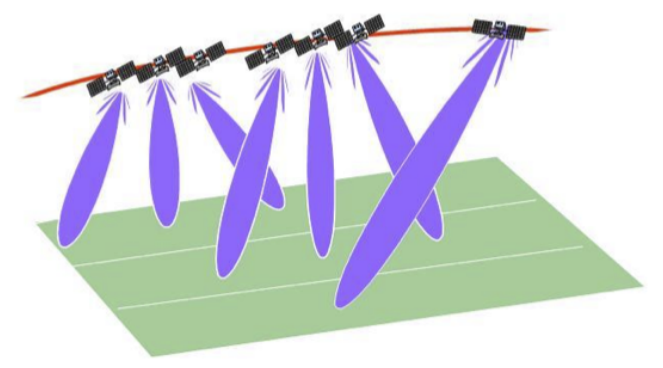

Figure 1. Schematic representation of the TOPSAR acquisition mode (from [5]). The beam is steered periodically in range, in order to cover several sub-swaths, and progressively in azimuth, aft to fore, in order to increase the azimuth bandwidth, expand the footprint and decrease scalloping. 


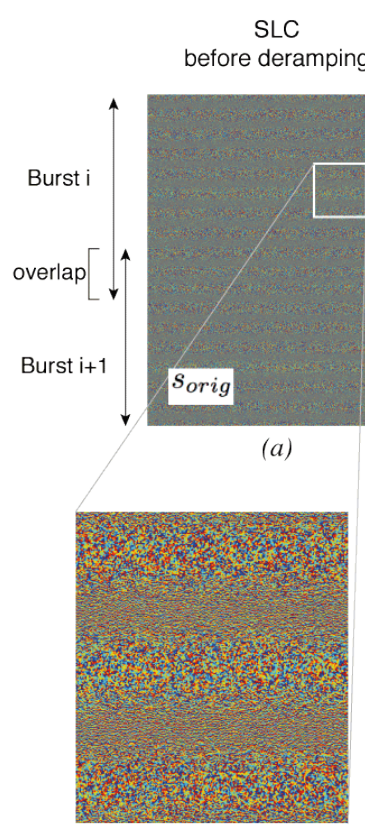

(d)

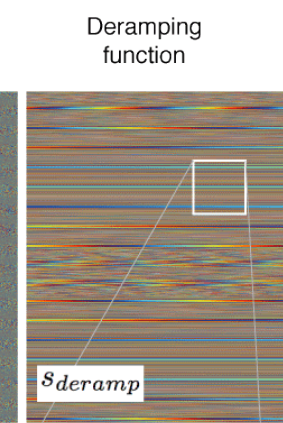

(b)

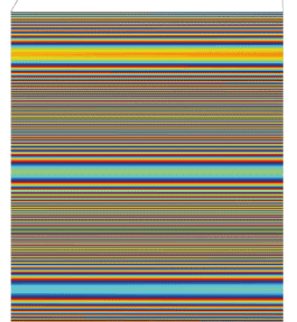

(e)
SLC

after deramping

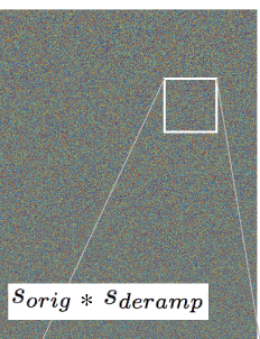

(c)

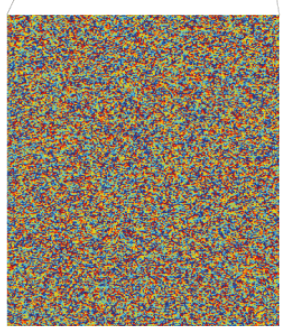

(f)

Figure 2. (a) Original phase of a Sentinel-1 TOPS SLC image. (b) Deramping function. (c) Corrected phase after multiplication by the deramping function. $(d)-(e)-(f)$ show zooms in the area outlined by the white square in $(a)-(b)-(c)$. For clarity, two successive bursts are shown over a small fraction of the swath width. The bursts are shown after stitching (i.e. burst overlap restored).

Here, a method for cancelling this phase term is presented. The method uses timing information relative to burst start and stop times that are usually provided with the image metadata, as well as additional information deduced from sub-pixel correlation between master and slave images. The output of the method is a set of two corrected images that can be subsequently ingested by a standard interferometric processing chain with only minimal modification. A particular emphasis is put on the implementation of the method to Sentinel-1 TOPS data provided in SLC format.

\section{IMPULSE RESPONSE FUNCTION}

The input SLC data are assumed to be preprocessed from raw data using a standard procedure for range compression, range cell migration correction (RCMC) and azimuth compression. Since the pre-processing treats every sub-swath independently, it is sufficient to describe the present method for the processing of a single sub-swath. Sub-swath stitching can be performed in post-processing.

Assuming the data are processed to zero Doppler, and neglecting amplitude modulation due to the antenna pattern in azimuth, the compressed target's response in the origi- nal focused SLC data is expressed as [5]:

$$
\begin{aligned}
s_{\text {orig }}(\eta, \tau)= & A \cdot \operatorname{sinc}\left(B_{e f f}\left(\eta-\eta_{0}\right)\right) \\
& \times \exp \left\{+j \pi k_{t}(\tau)\left(\eta-\eta_{c}(\tau)\right)^{2}\right\}
\end{aligned}
$$

where $A$ is a constant, $B_{\text {eff }}$ is the effective azimuth bandwidth that dictates the azimuth resolution, $k_{t}$ is the Doppler centroid modulation rate in the focused SLC data, $\tau$ is range (fast) time, $\eta$ is the azimuth (slow) time, $\eta_{0}$ is zero Doppler time of the target and $\eta_{c}$ is the burst center time. The exponential term results in the phase at the peak of the system's impulse response envelope (here, at $\eta=\eta_{0}$ ) being different from zero. The actually retrieved phase will therefore not only include the complex reflectivity response of the pixel, as well as its slant-range, but also an additional phase term that varies quadratically in azimuth. This azimuth phase term is evident in the Sentinel-1 SLC data provided by ESA (Figs. 2a. and 2d.).

The presence of this quadratic phase term poses a problem for interferometry, since the steep azimuth phase gradients resulting from a quadratic evolution of the phase are aliased over most of the duration of the burst, due to the sampling at the relatively low pulse repetition frequency (PRF). This issue is most serious in TOPS mode due to the large azimuth bandwidth, but it also arises in ScanSAR. Aliasing of the quadratic azimuth term leads to amplitude and phase distortions when resampling the slave image during coregistration with the master image. Amplitude and phase artefacts in the resulting interfero- 
grams are the signature of these distortions. Therefore, it is necessary to cancel the quadratic phase term prior to interferogram formation.

\section{PRINCIPLE OF DERAMPING}

In order to remove the azimuth quadratic phase term, the phase can be multiplied in the image (time) domain by an appropriate compensation term. This operation is referred to as "deramping". The deramping function is designed to cancel the quadratic drift, so as to bring the azimuth phase spectrum back to baseband:

$$
s_{\text {deramp }}(\eta, \tau)=\exp \left\{-j \pi k_{t}(\tau)\left(\eta-\eta_{\text {ref }}(\tau)\right)^{2}\right\}
$$

For Sentinel-1 TOPSAR (Fig. 1), the values of $k_{t}$ and $\eta_{c}$ can be deduced from the metadata following the guidelines provided in [6]. For our purposes, it suffices to mention that $\eta$ is the zero-Doppler azimuth time centered in the middle of the burst, i.e.:

$$
\eta=\left[-\frac{N_{b u}}{2} \cdot q,+\frac{N_{b u}}{2} \cdot q\right]
$$

where $q$ is the azimuth sampling interval, given as the inverse of the pulse repetition frequency $\left(q=\frac{1}{P R F}\right)$, and $N_{b u}$ is the number of samples is a burst. For Sentinel-1, $N_{b u}$ is often constant over a given sub-swath. Noting that the above expression is ambiguous as it yields different bounds for $\eta$ depending on the even or odd nature of $N_{b u}$, the following expression is used, instead:

$$
\eta=\left(l_{\eta}-\overline{l_{\eta}}\right) \cdot q \quad \text { with } \quad l_{\eta}=\left[1, N_{b u}\right] \in \mathbb{N}
$$

where $l_{\eta}$ is the line index relative to the first line within the current burst. The above expression implies that azimuth time $\eta$ is referenced to the burst mid-time $\eta_{\text {mid }}$ (i.e. $\eta=0$ for $\eta=\eta_{m i d}$ ), or, equivalently, to the burst start time $\eta_{\text {start }}$.

The reference time function $\eta_{r e f}$ is given by:

$\eta_{r e f}(\tau)=\eta_{c}(\tau)-\eta_{c}(0) \quad$ with $\quad \eta_{c}(\tau)=-\frac{f_{\eta c}(\tau)}{k_{a}(\tau)}$

where $\eta_{c}$ is the burst center time, $f_{\eta c}$ is the Doppler centroid frequency and $k_{a}$ is the "classical" Doppler FM rate (i.e. corresponding to the stripmap case). The functions $k_{t}(\tau), k_{a}(\tau)$ and $f_{\eta c}(\tau)$ are provided for every burst as a sequence of range polynomials in the metadata [6].

Deramping is an efficient method to reduce the quadratic phase term at first order. Unfortunately, due to inacurracies in Doppler centroid estimations, orbital parameters and DEM, perfect registration of a given target's location along the azimuth (sensing) time axis is never achieved in practice. As a consequence, the azimuth time coordinate of a given pixel will not exactly match from one acquisition to another. In other words, when two acquisitions are considered, the compressed response of a given ground target would actually be:

$$
s_{\text {orig }}^{s}(\eta, \tau)=s_{\text {orig }}^{m}\left(\eta-\Delta \eta_{\text {lag }}, \tau\right)
$$

where superscripts $m$ and $s$ refer to the master and slave acquisitions, respectively, and $\Delta \eta_{l a g}$ corresponds to the time lag between the target's illumination time in the slave acquisiton relative to the same time in the master image, with the time origins tied to the same location in space. This time lag is the result of miregistrations in azimuth time.

Should this time lag be incorrectly accounted for during deramping, a residual quadratic phase term would remain superimposed on the desired interferometric phase. This residual phase term corresponds to the difference between two slightly misaligned quadratic phase terms, which yields a linear phase ramp in azimuth expressed as:

$$
\phi(\eta, \tau=0)=2 \pi k_{t}(0) \Delta \eta_{l a g}\left(\eta+\frac{\Delta \eta_{l a g}}{2}\right)
$$

where $\tau=0$ has been assumed for simplicity. The residual azimuth phase slope is therefore proportional to the time lag $\Delta \eta_{\text {lag }}$ (Fig. 3). For values typical of Sentinel-1 IW mode $\left(k_{t}=1.7 \mathrm{kHz}, N_{b u}=1629\right.$, $q=2.055 \mathrm{msec}$ ), a misregistration error of 0.1 azimuth pixels (i.e. $\Delta \eta_{l a g}=0.1 * q$ ) induces a $2.4 \pi$ phase step at burst junction. Similar values were reported for TOPS interferometry with TerraSAR-X [7].

In order to assess and correct these misregistrations, an accurate method has been proposed by [7]. The method relies on exploitation of the spectral diversity in burst overlap regions, following the ideas introduced by [8]for the accurate coregistration of burst-mode data.

Alternatively, a more simple method is here introduced. Instead of using the phase information to achieve coregistration, it is possible to use the actual offset in the SLC images between the location of every target in the master and slave image. This offset can be determined by means of a sub-pixel correlation, which is a routine step in the standard procedure of interferometric calculation. As shown below, although less accurate than

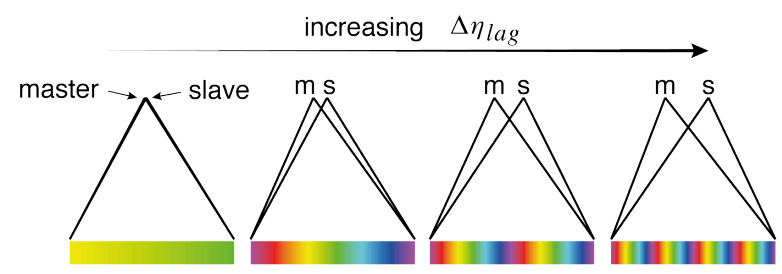

Figure 3. The slope of azimuth phase ramps induced by imperfect synchronization of master and slave acquisitions are proportional to the temporal lag between burst centre times in the two acquisitions. 
the Enhanced Spectral Diversity (ESD) method [7], the proposed method allows for substantially decreasing azimuth phase artefacts and thereby provide useful results for standard interferometric applications.

\section{PROPOSED METHOD}

\subsection{Step 1: burst stitching}

In the first step, the standard deramping function (Eq. 2) is applied to both master and slave images (Fig. 4). The multiplication by the deramping function is carried out for each burst separately.

The deramped bursts are then shifted by an appropriate number of azimuth samples $\Delta p$ so as to account for the azimuth overlap between successive bursts. Shifted

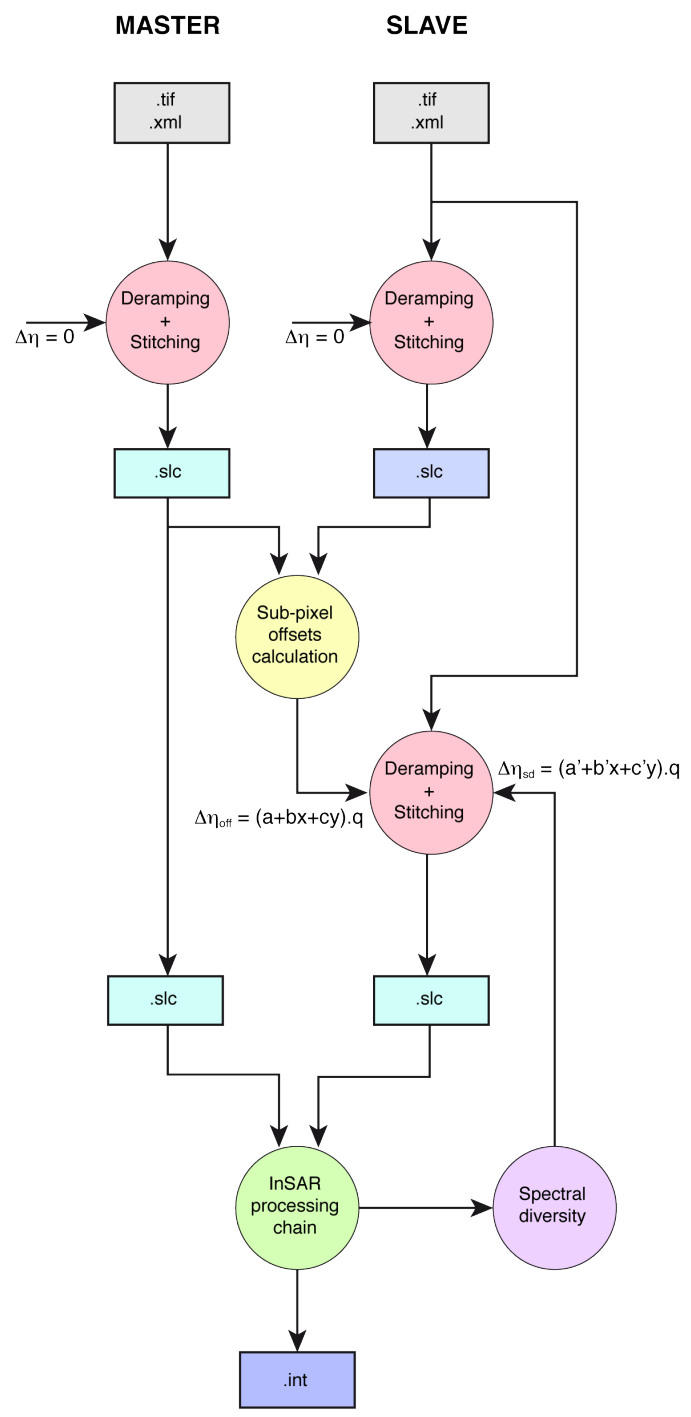

Figure 4. Workflow of the method. bursts are then mosaicked to produce a continuous image. This step is here termed "burst stitching". The azimuth shift $\Delta p^{i}$ for burst number $i$ is found by computing the difference between, on one hand, the number of azimuth pixels corresponding to the time difference between sensing of the first pulse of the current burst $\eta_{0}^{i}$ and sensing of the first burst in the previous burst $\eta_{0}^{i-1}$, and, on the other hand, the number of samples within current burst $N_{b u}^{i}$ :

$$
\Delta p^{i}=\left(\eta_{0}^{i}-\eta_{0}^{i-1}\right) / q-N_{b u}^{i}<0 \quad(\text { for } i>1)
$$

For Sentinel-1, the sensing time of the first line of any burst is provided in the metadata in two forms: either referenced to an absolute time reference (i.e. UTC time) or relative to the ascending node crossing time. Since the satellite evolves on a sun-synchronous orbit, any of these two times can be used, as only time differences during the course of the acquisition are relevant. We found $\Delta p^{i}$ to be close to an integer:

$$
\left|\Delta p^{i}-\operatorname{int}\left(\Delta p^{i}\right)\right| \approx 10^{-3}
$$

This characteristic of burst pacing stemps from the resampling of the data on a regular grid that is operated as part SLC generation [5]. A regular sampling implies that successive bursts can be, in good approximation, stitched by applying a simple translation by an integer number of azimuth samples. Therefore, a sophisticated resampling scheme is unnecessary at that stage.

We note that the number of pixels corresponding to the overlap region changes by a few pixels from one burst to another (i.e. $\Delta p^{i} \neq \Delta p^{j}$ for $i \neq j$ ), as well as from one acquisition to another for a given pair of consecutive bursts (i.e. $\Delta p^{m, i} \neq \Delta p^{s, i}$ ). In other words, the first line of burst number $i$ in the slave image does not necessarily correspond to the first line of burst number $i$ in the master image. As a consequence, the burst mid-times $\eta_{\text {mid }}$ that are used to reference the azimuth time $\eta$ in Eq. 4 will not match in general. This burst-to-burst and acquisition-toacquisition integer shift will be used in the next step to account for burst centre misalignment.

After appropriate shift in azimuth, burst overlap regions are split into two parts. The mid-time of the burst overlap region in the master image is used to place the burst separation for both the master and slave images. This way, the forward-looking phase is not mixed with the backwardlooking phase.

\subsection{Step 2: gross deramping using sub-pixel offsets}

In a second step, the actual time lag between the master and slave image is corrected by taking into account pixel offsets. After sub-pixel correlation of the slave image against the master image, an affine transformation is determined by least-squares fitting of the pixel offsets (Fig. 5). The affine transformation consists in translating pixels with coordinates $(x, y)$ in the original slave image 

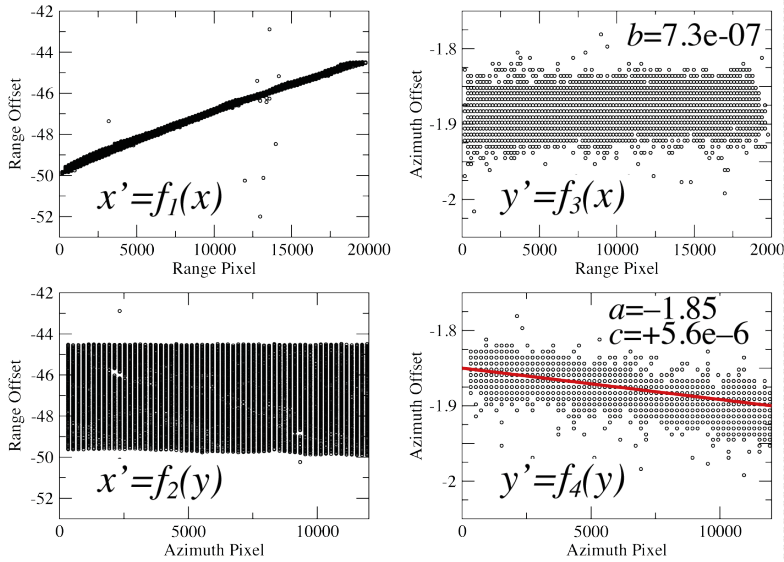

Figure 5. Offsets between the master and slave image measured from incoherent cross-correlation. The left panels correspond to the range offsets, while the right panels correspond to the azimuth offsets. The upper panels show the offsets as a function of the range coordinate within the master image, whereas the lower panels represent the same offsets as a function of azimuth coordinate. The red line shows the fitted variation of the azimuth offset as a function of azimuth in the affine transformation applied during coregistration. The slope indicates an azimuth stretching of the slave image with respect to the master image.

to a new location given by coordinates $\left(x^{\prime}, y^{\prime}\right)$ in the resampled slave image. The coordinates are related according to the matrix operation:

$$
\left[\begin{array}{l}
x^{\prime} \\
y^{\prime}
\end{array}\right]=\left[\begin{array}{ll}
A & B \\
D & E
\end{array}\right]\left[\begin{array}{l}
x \\
y
\end{array}\right]+\left[\begin{array}{l}
C \\
F
\end{array}\right]
$$

Since only the azimuth translation is of interest here, the azimuth time offset $\Delta \eta_{o f f}$ associated with the affine transformation is given by:

$$
\begin{array}{r}
\Delta \eta_{o f f}(x, y)=\left(y^{\prime}-y\right) \cdot q=(a+b x+c y) \cdot q \\
\text { with }\left\{\begin{array}{llc}
a= & F \\
b= & D \\
c= & (E-1)
\end{array}\right.
\end{array}
$$

At this point, the time coordinates $\eta$ are not yet synchronized. Indeed, due to variations in the length of the burst overlap regions, the mid-time of any burst in the master image $\eta_{m i d}^{m}$ does not exactly match with the mid-time of the corresponding burst in the slave image $\eta_{m i d}^{s}$. Indeed, as mentioned above, the burst sensing start time of a given burst $\eta_{\text {start }}^{i}$ generally differs by an integer number of azimuth samples between the slave and master images (Eq. 8). The initial time difference at the beginning of the image acquisition, $\eta_{\text {start }}^{s, 1}-\eta_{\text {start }}^{m, 1}$, can be understood as the predicted offset at the top of the image. However, due to the regular sampling of the SLC, this predicted offset should only change by an integer number of azimuth pixels across burst transitions, and otherwise remain constant in burst interiors. In contrast, computation of actual offsets between images by means of sub-pixel correlation demonstrates that the images are slightly distorted. In order to account for these distortions, an additional term is added (Fig. 6):

$$
\Delta \eta_{\text {shift }}^{i}=\left(\eta_{\text {start }}^{s, 1}-\eta_{\text {start }}^{m, 1}\right)-\left(\eta_{\text {start }}^{s, i}-\eta_{\text {start }}^{m, i}\right)
$$

In Eq. 12, the second term in braces corresponds to the time difference between burst sensing start time for the current burst $i$ in slave and master images. The first term is introduced to account for the initial time difference at the beginning of the image acquisition, i.e. the predicted time offset at the top of the image. The above formula allows for cancelling this predicted offset throughout the image, which will be replaced by the measured offset $\Delta \eta_{\text {off }}$.

Finally, the full expression of the deramping function that is applied to the slave image reads:

$$
\begin{gathered}
s_{\text {deramp }}^{\prime}(\eta, \tau)=\exp \left\{-j \pi k_{t}(\tau)\left(\eta-\Delta \eta_{\text {lag }}-\eta_{\text {ref }}(\tau)\right)^{2}\right\} \\
\Delta \eta_{\text {lag }}=\Delta \eta_{\text {shift }}+\Delta \eta_{\text {off }}
\end{gathered}
$$

Using this expression, burst deramping and stitching is again applied to the original slave SLC image (Fig. 4).

The master and slave images are then ready for interferometric processing. At this stage, residual phase ramps are significantly reduced in the corrected interferograms. This demonstrates that the accuracy of the misregistration error, which depends on the quality of sub-pixel off-

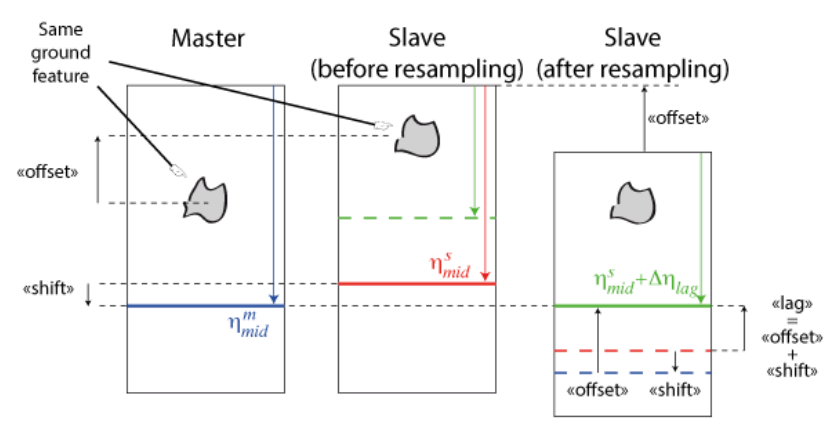

Figure 6. Sketch showing the spatial / temporal relationship between the centre times $\eta_{\text {mid }}$ of one burst as seen in the master and slave images. In the master image, the centre time $\eta_{m i d}^{m}$ is referenced to a time origin that differs from the time origin used to index the centre time in the slave image $\eta_{\text {mid }}^{s}$. This difference corresponds to the offset between the two origin times, which can be resolved by means of a sub-pixel correlation. Furthermore, the burst mid-time in the master image does not correspond to the same azimuth line as the burst centre time in the slave image, resulting in an additional shift by an integer number of azimuth lines between the two burst centres. The sum of these two tems (offset and shift) yields the appropriate time lag that should be included in the deramping function of the slave image so as to compensate for the slightly different time origins. 
sets determined from the amplitude image, is already relatively good. Accordingly, the performance of the subpixel correlation depends on surface conditions.

\subsection{Step 3: refined deramping using spectral diver- sity}

Although the corrected images already yield interferograms with a reasonable quality, the above method is still insufficiently accurate for high-precision applications, such as monitoring of slow tectonic deformation. Imperfection of the coregistration can be observed in places where sub-pixel offsets have yielded ambiguous results, resulting in a poor estimation of the affine coefficients. These residual errors are manifested as phase jumps across burst boundaries.

These phase jumps can be interpreted as the difference, in slant-range time, between on one hand, the forwardlooking sensor-to-ground slant range just before (above) a given burst boundary, and on the other hand, the backward-looking slant-range just after (below) the burst boundary. In case of significant ground displacements between two diachronic acquisitions, this difference is proportional to the ground motion in the azimuth direction. This is the concept behind the so-called "multipleaperture" interferometry method, which can be applied by splitting the beam in two parts in the Stripmap mode [9].

Alternatively, it is possible to achieve a precise coregistration by exploiting the proportionality between the pixel offset and the residual phase difference, according to the so-called "spectral diversity" method [8, 7]. In burstmode, this difference between the forward and backward interferometric phases is due to the squint difference in burst overlap regions, which equals $\sim 2^{\circ}$ for Sentinel1. Since the forward- and backward-looking phases are both provided in the original SLC product, this difference can be readily measured after multilooking the crossinterferogram (Fig. 7.a). The phase difference $\Delta \phi_{s d}$ is related to the misregistration error $\Delta \eta_{s d}$ according to:

$$
\Delta \phi_{s d}=2 \pi \Delta f_{\text {ovl }} \Delta \eta_{s d} \quad \bmod [2 \pi]
$$

where $\Delta f_{\text {ovl }}$ is the Doppler frequency difference in the burst overlap region. This frequency difference can be deduced from the azimuth distance $\Delta p_{\text {ovl }}$ between the middle positions of two successive bursts, counted in number of pixels:

$$
\Delta f_{o v l}=\overline{k_{t}} \Delta p_{o v l} q \quad \text { with } \quad \Delta p_{o v l}=N_{b u}+\frac{N_{o v l}}{2}
$$

where $\overline{k_{t}}$ stands for the Doppler centroid modulation rate averaged over the image and $N_{o v l}$ is the number of azimuth pixels in a burst overlap region (typically $\sim 10 \%$ of the burst length $N_{b u}$ ). In practice, the phase retrieved in the cross-interferogram $\Delta \phi_{s d}$ is observed to vary slowly across the image. A first order polynomial surface (i.e. a straight plane) can be used to fit the retrieved phase (a)

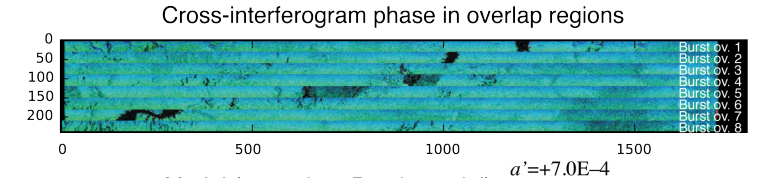

(b)

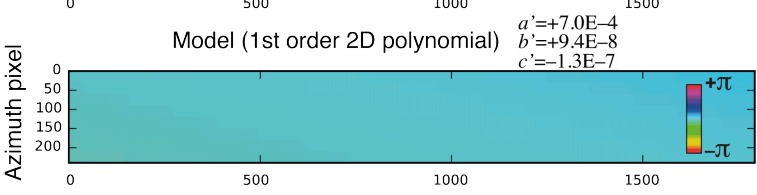

(c)

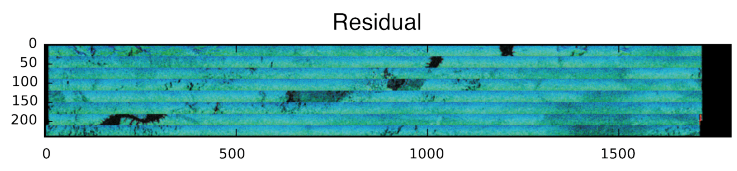

Figure 7. Refinement of the lag estimation using spectral diversity. The top row shows the phase retrieved in the cross-interferogram over eight successive burst overlap regions. For clarity, the phase in non-overlap regions is not shown. The middle row shows the first-order polynomial surface adjustment. The bottom row shows the residual.

(Fig. 7.b). This way, the affine coefficients in Eq. 11 can be refined by accounting for the residual lag deduced from spectral diversity:

$$
\Delta \eta_{s d}(x, y)=\left(a^{\prime}+b^{\prime} x+c^{\prime} y\right) \cdot q
$$

Accordingly, a higher order polynomial surface could be used to improve the fit to the observed phase evolution along azimuth and range. Nevertheless, the linearity of Eq. 16 has the advantage of providing suitable conditions for combining a large number of images within a network of interferograms without requiring that every pair should be computed.

Finally, the lag term in the deramping function (Eq. 13) is updated using the refined estimate provided by spectral diversity:

$$
\Delta \eta_{l a g}^{\prime}=\Delta \eta_{l a g}+\Delta \eta_{s d}
$$

The slave SLC is deramped a third time using the above expression, and the interferogram can be recalculated (Fig. 7.c).

An example of the resulting interferograms obtained using the proposed method is presented in Fig. 8. Phase jumps across burst boundaries, as well as across subswaths, are significantly reduced.

\section{CONCLUDING REMARKS}

This study demonstrates that, despite the rule-of-thump accuracy of 0.1 pixel achieved by the sub-pixel correlation method, it is possible to derive a misregistration error with sufficient accuracy to substantially reduce residual phase ramps. This suggests that the large support enabled by the correlation estimation performed across the full image allows for determining affine coefficients in 


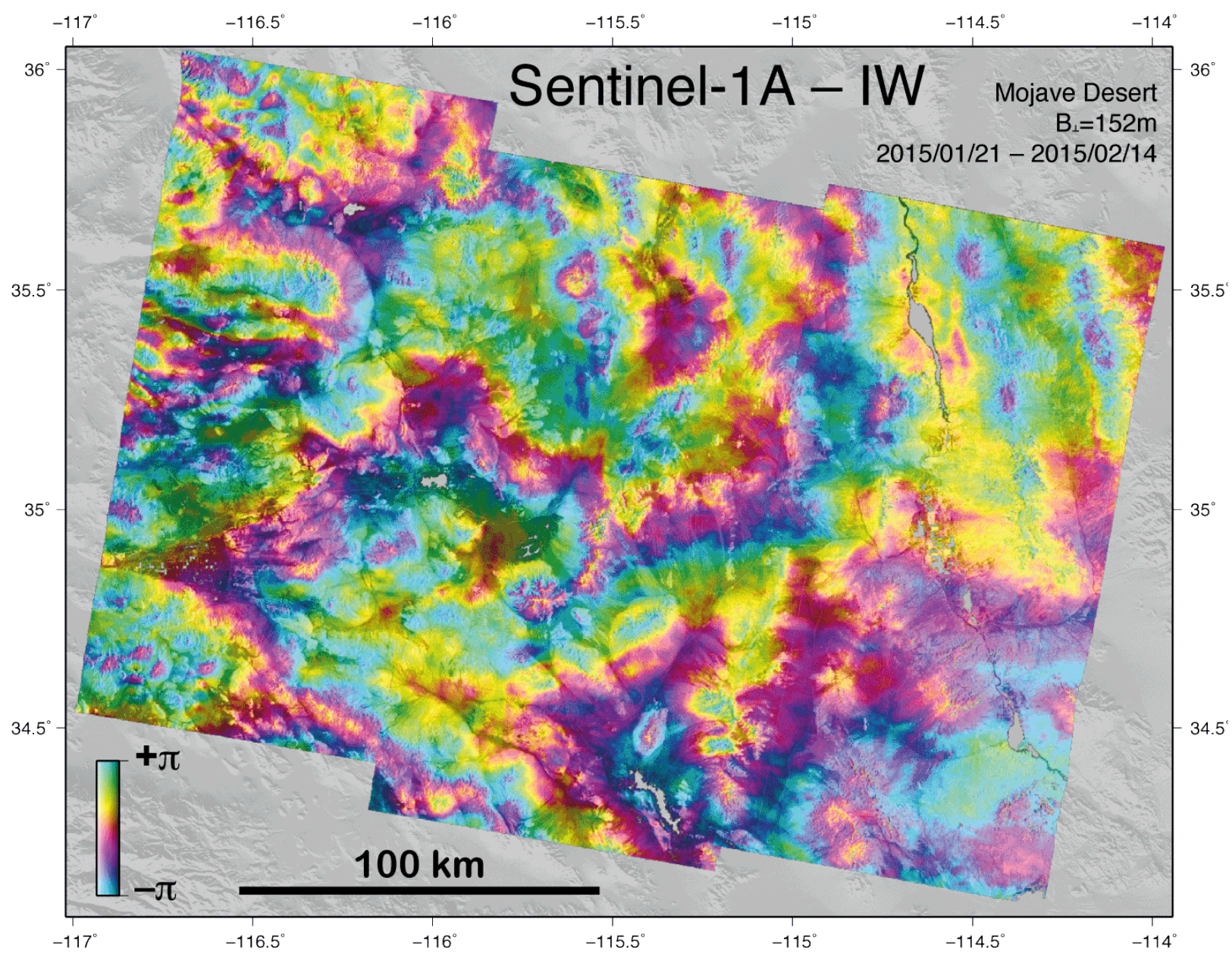

Figure 8. Sentinel-1A TOPS interferogram computed with the proposed method. The interferogram covers a highly coherent area in the Mojave desert, western USA.

Eq. 10 with sufficient accuracy to derive the actual offset induced by image misregistration with a much better accuracy than achieved on a single correlation window. Furthermore, the short revisit time of Sentinel-1 should guarantee that little surface changes occur in practice, leading to good coherence and reliable sub-pixel offset retrievals. In spite of being less accurate than the ESD method, the proposed method has the advantage of simplicity and reasonable efficiency. Accordingly, the accuracy of the method depends on the quality of the retrieved offset field, but also relies on the high percentage of burst synchronization achieved by the Sentinel-1 system.

\section{ACKNOWLEDGMENTS}

This work has been supported by CNRS-PNTS (grant $\mathrm{n}^{\circ}$ PNTS-2015-09).

\section{REFERENCES}

[1] Andrea Monti-Guarnieri and Pietro Guccione. Optimal "focusing" for low resolution ScanSAR. Geoscience and Remote Sensing, IEEE Transactions on, 39(3):479-491, 2001.

[2] Francesco De Zan and A Monti Guarnieri. TOPSAR: Terrain observation by progressive scans. Geoscience and Remote Sensing, IEEE Transactions on, 44(9):2352-2360, 2006.

[3] Ian G Cumming and Frank Hay-chee Wong. Digital processing of synthetic aperture radar data: algorithms and implementation. Artech House, 2005.

[4] Pau Prats, Rolf Scheiber, Josef Mittermayer, Adriano Meta, and Alberto Moreira. Processing of sliding spotlight and TOPS SAR data using baseband azimuth scaling. Geoscience and Remote Sensing, IEEE Transactions on, 48(2):770-780, 2010.

[5] MDA. Sentinel-1 Level 1 Detailed Algorithm Definition. Technical Report S1-TN-MDA-52-7445, European Space Agency, 2014.

[6] Nuno Miranda. Definition of the TOPS SLC deramping function for products generated by the S-1 IPF. Technical Report COPE-GSEG-EOPG-TN-140025, European Space Agency, 2014.

[7] Pau Prats-Iraola, Rolf Scheiber, Luca Marotti, Steffen Wollstadt, and Andreas Reigber. TOPS interferometry with TerraSAR-X. Geoscience and Remote Sensing, IEEE Transactions on, 50(8):3179-3188, 2012.

[8] Rolf Scheiber and Alberto Moreira. Coregistration of interferometric SAR images using spectral diversity. Geoscience and Remote Sensing, IEEE Transactions on, 38(5):2179-2191, 2000.

[9] Noa BD Bechor and Howard A Zebker. Measuring two-dimensional movements using a single insar pair. Geophysical Research Letters, 33(16), 2006. 